\title{
Angiotensin receptor blockers: do they protect against dementia and Alzheimer's disease in the elderly?
}

\author{
Tzvi Dwolatzky ${ }^{\dagger}$
}

Evaluation of: Li NC, Lee A, Whitmer RA et al.: Use of angiotensin receptor blockers and risk of dementia in a predominantly male population: prospective cohort analysis. BMJ 340, B5465 (2010). A large observational study with a relatively long period of follow-up was designed to evaluate whether angiotensin receptor blockers are associated with protection against Alzheimer's disease or dementia. In the almost entirely male study population (mean age: 74 years) with cardiovascular disease, the use of angiotensin receptor blockers was associated with a reduced incidence of Alzheimer's disease and dementia. For patients with these conditions, the use of angiotensin receptor blockers reduced both mortality and the rate of admission to a nursing home. While these results are of great interest, they should be interpreted with great caution, since the findings refer to a rather specific study population and, thus, lack generalizability. Further research involving more heterogeneous samples is necessary.

In a recent issue of the British Medical Journal, $\mathrm{Li}$ and colleagues report the results of a study designed to evaluate whether angiotensin receptor blockers exert a protective effect on the incidence and progression of Alzheimer's disease (AD) and dementia [1] . The rationale for the study is based on the importance of the renin-angiotensin system in controlling hypertension and decreasing cardiovascular morbidity. Renin is produced in the kidneys and acts to cleave the peptide bond between leucine and valine on angiotensinogen, forming the peptide angiotensin I, which apparently has no biological activity. This is then converted by the angiotensin-converting enzyme (ACE), found predominantly in the lung, to angiotensin II, which binds to receptors and results in vasoconstriction, the synthesis and secretion of aldosterone, and endothelial proliferation, as well as leading to the development of atherosclerosis [2]. ACE inhibitors are effective in lowering blood pressure by their effect on preventing the formation of angiotensin II. The angiotensin II receptor type 1 (AT1) is the receptor that has been most studied.

Angiotensin receptor blockers act on AT1 to lower blood pressure. They have been found to exert cardioprotective effects and are superior in protecting against diabetes mellitus [3] and stroke [4]. It has been suggested that the inhibition of the renin-angiotensin system may represent a new treatment option for $\mathrm{AD}$ [5]. In addition, the finding of vascular changes in the brains of patients with AD has been well described [6]. Thus, the investigation of the possibly protective effect of angiotensin receptor blockers on the development of dementia is both interesting and relevant.

\section{Summary of methods \& results}

The study by Li et al. involved examining the records of approximately 7.3 million people in the database of the Veterans Health System over a period of 5 years. Patients aged 65 years or older with cardiovascular disease were studied. Three cohorts were examined, namely those taking angiotensin receptor blockers, those taking the ACE inhibitor lisinopril, and a comparative group taking cardiovascular drugs other than either these two classes, or statins. The time to the onset of incident $\mathrm{AD}$ or dementia over a 4-year period for those who were initially free of these conditions was determined. Outcome measures for the progression of $\mathrm{AD}$ and dementia were defined as admission to a nursing home or death. A total of 819,491 patients with AD and 799,069 with dementia were studied. The characteristics of these groups and the therapeutic cohorts were similar, having a mean age of 74 years and with $98 \%$ being men. There were significant differences between therapeutic groups with regard to comorbid conditions, with cardiovascular disease and stroke being more prevalent in the cardiovascular comparator group, and diabetes being more prevalent in the angiotensin

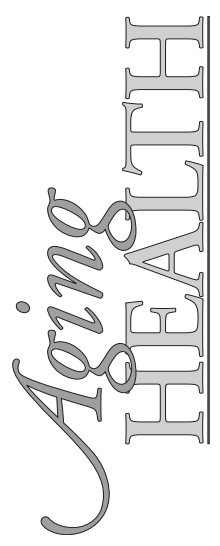

${ }^{\dagger}$ Department of Geriatrics \& Memory Clinic, Mental Health Center, PO Box 4600, Beersheva 84170 , Israel

Tel.: +97286401765

Fax: +972 86401565

tzvidov@bgu.ac.il

\section{Keywords}

- Alzheimer's disease

- angiotensin receptor blocker

- cognition • dementia • elderly

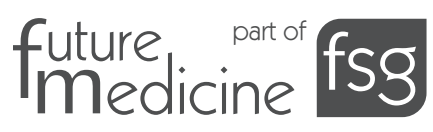


receptor blocker and lisinopril groups, as could be expected. There were no differences in blood pressure between groups.

Cox proportional hazard models were adjusted for age, diabetes, stroke and cardiovascular disease. Compared with the cardiovascular comparator group, the hazard ratio for incident dementia in the angiotensin receptor blocker group was 0.76 (95\% CI: 0.69-0.84), and 0.81 (95\% CI: 0.73-0.90) compared with the lisinopril group. For those with $\mathrm{AD}$, the use of angiotensin receptor blockers was associated with a significantly lower risk of admission to a nursing home (HR: $0.51 ; 95 \% \mathrm{CI}: 0.36-0.72$ ) and death (HR: 0.83 ; 95\% CI: $0.71-0.97$ ) compared with the cardiovascular comparator group. There was a dose-response effect of angiotensin receptor blockers on the incidence of dementia. The combination of angiotensin receptor blockers with ACE inhibitors exhibited an additive effect, both on incident $\mathrm{AD}$ and dementia and on nursing home admission.

\section{Discussion}

The growing number of older people developing dementia places an increasing burden on families and healthcare services. The chronic nature of this condition results in the utilization of community and institutional resources for extended periods, frequently at a high economic cost. The etiology of dementia is most often related to neurodegenerative conditions, with $\mathrm{AD}$ being the most common. Among the important risk factors for dementia are age, female sex, previous significant head trauma, ApoE4 genotype and low education level. Cardiovascular risk factors, including hypertension, hyperlipidemia, diabetes mellitus and smoking, are considered to be important potentially treatable causes of age-related cognitive impairment and dementia. In addition, the identification of agents that are capable of exerting effects preventing vascular and neurodegenerative changes in the aging brain will probably have major ramifications on healthcare.
The results of this large, well-designed study may have important therapeutic consequences for the increasing number of older people suffering from $\mathrm{AD}$ and other dementias. These conditions place a significant economic and emotional burden on caregivers in particular, and on society in general. The lack of effective treatments for these conditions at the present time makes the search for other possible agents and interventions to prevent and alleviate cognitive decline and dementia all the more pressing. The clear benefit demonstrated in this study of angiotensin receptor-blocking drugs, both on the development of incident dementia and on subsequent nursing home placement and mortality, is of great interest. While this finding confirms the results of previous smaller studies demonstrating the beneficial association between angiotensin receptor blockers and cognitive function, there are a number of limitations to this study that should be emphasized. The use of a population-derived database raises the possibility of misclassification of disease, in particular that of $\mathrm{AD}$ and dementia, as well as other undetermined biases. In addition, the study of male veterans limits the generalizability of the findings of this study to other population groups. The study did not evaluate possible adverse effects of the therapeutic interventions in each group, and the knowledge of such effects is essential in determining the true benefit of these compounds.

The biological basis for these findings is of great interest. The authors emphasize the strong association of stroke with dementia and nursing home admission found in this study. However, this is probably not related to blood pressure control, which was similar between groups. In fact, the association between cardiovascular risk factors and $\mathrm{AD}$ has been questioned recently, when a systematic review found that clinically defined risk factors, such as hypertension and diabetes, were not significantly associated with an increased risk of $\mathrm{AD}$ [7]. The role of the angiotensin receptor blockers on cerebral blood

\section{Executive summary}

- Dementia, the most common cause of which is Alzheimer's disease (AD), is highly prevalent among the older population in developed countries.

- Dementia places a significant burden on caregivers and on healthcare services.

- Effective therapeutic interventions for dementia and AD are limited.

- The search for agents with protective or beneficial effects in these conditions is of the utmost importance.

- Angiotensin receptor blockers may protect against the development of AD and dementia, and may decrease mortality and delay nursing home placement in patients with these conditions.

- Further studies will clarify whether these agents are beneficial in preserving cognitive function. 
flow and their possible neuroprotective effects have been described; however, these and other possible mechanisms need to be studied further.

\section{Future perspective}

The role of angiotensin receptor blockers in preventing cognitive decline and the progression of dementia, including AD, is strengthened by this study. A well-designed randomized controlled study involving a heterogeneous population sample should follow, with emphasis placed on the definition of AD and dementia, as well as cognitive outcome measures. The possible mechanisms of action of angiotensin receptor blockers on cerebral function and neurobiology should be further elucidated.

\section{Financial \& competing interests disclosure}

The author has no relevant affiliations or financial involvement with any organization or entity with a financial interest in or financial conflict with the subject matter or materials discussed in the manuscript. This includes employment, consultancies, honoraria, stock ownership or options, expert testimony, grants or patents received or pending, or royalties. No writing assistance was utilized in the production of this manuscript.

\section{Bibliography}

1. Li NC, Lee A, Whitmer RA et al.: Use of angiotensin receptor blockers and risk of dementia in a predominantly male population: prospective cohort analysis. BMJ 340, B5465 (2010).

2. Sata M, Fukuda D: Crucial role of reninangiotensin system in the pathogenesis of atherosclerosis. J. Med. Invest. 57, 12-25 (2010).
3. Andraws R, Brown DL: Effect of inhibition of the renin-angiotensin system on development of Type 2 diabetes mellitus (meta-analysis of randomized trials). Am. J. Cardiol. 99, 1006-1012 (2007).

4. Lu GC, Cheng JW, Zhu KM, Ma XJ, Shen FM, Su DF: A systematic review of angiotensin receptor blockers in preventing stroke. Stroke 40, 3876-3878 (2009).

5. Kehoe PG, Wilcock GK: Is inhibition of the renin-angiotensin system a new treatment option for Alzheimer's disease? Lancet Neurol. 6, 373-378 (2007).
6. Launer LJ, Petrovitch H, Ross GW, Markesbery W, White LR: AD brain pathology: vascular origins? Results from the HAAS autopsy study. Neurobiol. Aging 29, 1587-1590 (2008).

7. Purnell C, Gao S, Callahan CM, Hendrie HC: Cardiovascular risk factors and incident Alzheimer disease: a systematic review of the literature. Alzheimer Dis. Assoc. Disord. 23, 1-10 (2009). 